\title{
The radiation burden from increasingly complex endovascular aortic aneurysm repair
}

\author{
Avnesh S. Thakor - Andrew Winterbottom • \\ Mathew Mercuri • Claire Cousins • Michael E. Gaunt
}

Received: 25 April 2011 /Revised: 27 May 2011 /Accepted: 12 July 2011 /Published online: 31 July 2011

(C) European Society of Radiology 2011

\begin{abstract}
Objectives With increasing experience, endovascular aortic aneurysm repair (EVAR) has been extended to patients with less suitable aorto-iliac anatomy in an attempt to reduce peri-operative mortality. However, more complex EVAR procedures may take longer and can result in higher rates of complications, additional interventional procedures and more frequent radiological imaging, which may offset some of the benefit. This study determined the radiation burden for standard EVAR, as determined by the EVAR-1 trial criteria, and more complex EVAR.

Methods A total of 123 elective patients aged $>60$, with aneurysms $>5.5 \mathrm{~cm}$ who received a bifurcated stent-graft were allocated into a group based on whether or not they fulfilled strict EVAR-1 trial criteria. The mean radiation dose was calculated for each group, together with the additional radiation burden from routine pre- and post-EVAR CT examinations and pre-EVAR iliac artery embolisation.

Results Patients not meeting the EVAR-1 trial criteria had significantly longer fluoroscopic screening times and higher radiation doses. The radiation burden in all patients was
\end{abstract}

A. S. Thakor · A. Winterbottom $\cdot$ C. Cousins

Department of Radiology, Addenbrookes Hospital,

University of Cambridge,

Cambridge, UK

\section{E. Gaunt ( $\square)$}

Department of Vascular Surgery, Addenbrookes Hospital,

University of Cambridge,

Cambridge CB2 2QQ, UK

e-mail:meg34@cam.ac.uk.

\section{Mercuri}

Health Research Methodology, Hamilton Health Sciences,

McMaster University,

Hamilton, Canada higher following exposure from routine CT examinations and following pre-EVAR iliac artery embolisation.

Conclusion Whilst the radiation from standard EVAR is acceptable, more complicated and challenging EVARs, accompanied with additional radiological investigations and procedures, can significantly increase the radiation burden.

Keywords Endovascular · Aortic aneurysm · Repair . Radiation risk

\section{Introduction}

In the early 1990s, endovascular aortic aneurysm repair (EVAR) was introduced as an alternative to open repair for the treatment of aortic aneurysms. To assess the effectiveness of treating patients with EVAR compared with open aneurysm repair, two large randomized multicentre trials (EVAR-1 [1] and DREAM [2] Trials) were undertaken between 1999 and 2003. In the EVAR-1 Trial, patients were selected based on their fitness to undergo surgery and specific criteria relating to aortic aneurysm morphology considered favourable for EVAR. The results were published in 2004 and revealed that patients who underwent EVAR, but who were fit enough for open aneurysm repair, had fewer post-procedural complications, a reduced hospital stay and a 3\% reduction in 30-day mortality $(1.7 \%$ vs $4.7 \%)$ [1]. Furthermore, at 4 years of follow-up, the EVAR-1 Trial also demonstrated a persistent reduction in aneurysm-related mortality in EVAR patients compared with those who received open repair (4\% vs $7 \%$ ) [3].

Recently, there has been increasing concern regarding the radiation risks to both the patient [4] and operator [5] for fluoroscopically based interventional procedures. Indeed, 
dose area product (DAP) values have been reported as high as 125,338 and 459 Gy.cm ${ }^{2}$ for cardiac-, neuro- and intravascular abdominal-interventional procedures, respectively $[6,7]$. However, there is a certain inconsistency within the literature for the average radiation dose a patient receives from an EVAR procedure, with values ranging from 43 to $150 \mathrm{~Gy} . \mathrm{cm}^{2}[8-10]$. This has resulted in uncertainty amongst interventional radiologists as to the potential long-term effects which these doses of radiation may be having on patients. This is further compounded by the number of computerised tomography (CT) examinations undertaken both before and after EVAR; each of which exposes the patient to a further dose of ionizing radiation. Indeed, a recent study estimated the average EVAR patient undergoes five CT examinations with intravenous contrast medium within their first 2 years [11]. In addition, clinicians are also undertaking increasingly complex EVAR procedures in patients with less favourable aortic and iliac anatomy, which require longer fluoroscopic screening times. Many of these procedures also require additional radiological interventions such as pre-EVAR occlusion of internal iliac arteries and post-operative embolisation/coiling for type II endoleaks associated with aneurysm sac expansion. These more complex EVAR procedures are also more likely to develop stent related complications, such as proximal type I endoleaks and graft limb dislocations, which again will require further evaluation with $\mathrm{CT}$ and/or angiograms followed by correction with re-EVAR procedures. Furthermore, there is also a trend to now advocate EVAR in younger patients who have both a low risk for an open repair [12] and a greater lifetime risk of developing cancer following radiation exposure.

One explanation for the inconsistency in the radiation dose received by patients undergoing EVAR could be the range of complexity of the procedure within the groups being analysed. Hence, the present study retrospectively divided patients who received a bifurcated aortic stent graft into two groups, depending on the complexity of the EVAR procedure as determined by the anatomical criteria for the EVAR-1 Trial, with those patients meeting the criteria having aneurysms deemed to be less complicated. We hypothesize patients who have more complex aortic anatomy will have more complicated procedures with longer fluoroscopic screening times and higher doses of radiation.

\section{Materials and methods}

Patient selection

Our unit has been performing EVAR since 2002 and was a participant in the EVAR-1 Trial. A consultant interventional radiologist and consultant vascular surgeon jointly performed each EVAR procedure. In the present study, radiation dose data was retrospectively collected for all patients who underwent elective EVAR in our tertiary referral vascular unit. Of the 203 patients in our database, only 123 were selected for the analysis in the present study since these patients were suitable candidates for either elective open or endovascular repair, aged over 60 , had an abdominal aortic aneurysm diameter $>5.5 \mathrm{~cm}$ and underwent an elective EVAR with a bifurcated aortic stent. In addition, radiation doses were collected for all patients who underwent pre-EVAR iliac artery embolisation.

\section{Patient subgroups}

Each patient had their pre-procedural CT examination re-reviewed by a consultant vascular radiologist, who allocated them to a group based on whether or not they had an aorta which fulfilled all the criteria required for them to be entered into the EVAR-1 Trial. The inclusion criteria included all of the following: a neck length $>15 \mathrm{~mm}$, neck outer diameter $<32 \mathrm{~mm}$, angulation in any plane $<60^{\circ}$, flare $<4 \mathrm{~mm}$ in $1 \mathrm{~cm}$, distal aorta diameter $>20 \mathrm{~mm}$, common iliac artery diameter $<21 \mathrm{~mm}$, external iliac artery $>6 \mathrm{~mm}$ and a subjective impression of non-tortuous iliac arteries.

\section{Data collection}

All data was collected retrospectively as part of a medical audit and hence ethical approval was not required. For each patient, their age, gender, fluoroscopic screening time and the dose of radiation received during the EVAR procedure was collected from information electronically stored on our picture archiving and communications system (PACS). All patients received one CT examination pre-EVAR followed by two surveillance CT examinations at 6 weeks and 12 months post-EVAR (as per the EVAR-1 Trial protocol). The initial planning CT was used to determine the anatomy of the patient's aorta in order to correctly size the aortic stent and determine the optimum landing zone, whilst the surveillance CT examinations were to check for any complications following stent placement. The average radiation dose received from a $\mathrm{CT}$ aorta examination was calculated from the parameters set in the $\mathrm{CT}$ protocol.

\section{Radiation measurements}

All EVAR procedures were performed in an operating theatre using continuous fluoroscopy with a mobile digital C-arm X-ray machine (Siemens Siremobile Compact L, Siemens UK, Surrey, UK), with image intensifier sizes of 17 and $23 \mathrm{~cm}$ and a filtration of $3 \mathrm{~mm}$ aluminium. Imaging was posterior-anterior with the X-ray tube (Maquet Alphamaxx, 
Maquet, Rustatt, Germany) placed below the operating table. Radiation doses were measured in terms of Dose Area Product (DAP; Gy. $\mathrm{cm}^{2}$ ), by a built-in ionisation chamber, calibrated by an independent DAP-meter (VacuDAP 2000, VacuTec Meßtechnik, Dresden, Germany) and regularly serviced every 6 months by the manufacturer. The reproducibility of DAP readings was approximately $\pm 1.5 \%$. Pre-EVAR iliac artery embolisation procedures were performed in a dedicated interventional fluoroscopy suite.

Planning and surveillance CT examinations were performed using one of two CT systems (Siemens Sensation 16 and Siemens Sensation 64, Siemens Medical, Bracknell, UK), using the same standardised aorta protocol, with 5-mm unenhanced slices and 1-mm slices following intravenous contrast medium (all at $120 \mathrm{kV}$ with modulation of the tube current with respect to patient size: approx 120-160 mA). Radiation doses were measured in terms of dose length product (DLP; $\mathrm{mGy} . \mathrm{cm}$ ) and were automatically recorded at the time of CT data acquisition based on the $\mathrm{CT}$ parameters and independently verified using a $10 \mathrm{~cm}$ long pencil ionisation chamber (Radcal 20X6-3CT, Radcal corporation, Monrovia, CA, USA).

From the DAP data, the total body effective dose (ED), measured in milli-Sieverts (mSv), for the average size patient was calculated using a verified Monte Carlo computer program (PCXMC 1.5; STUK; Radiation and Nuclear Safety Authority, Helsinki, Finland). The PCXMC 1.5 based its calculations for EVAR on a focus to skin distance of $30 \mathrm{~cm}$, tube voltage of $90 \mathrm{kVp}$ and beam area of $254 \mathrm{~cm}^{2}$. From the DLP data, the ED in $\mathrm{mSv}$ was calculated based on methods described by Huda and colleagues (conversion factor $=0.0175$ ) [13]. Both the DAP and DLP were converted to $\mathrm{mSv}$ in order for the cumulative dose of radiation to be calculated for analysis.

\section{Data and statistical analysis}

Values for all variables were expressed as the mean \pm SEM. All measured variables were first analysed for normality of distribution and then assessed using an unpaired $t$-test comparing the effect of aortic anatomy complexity as determined by the EVAR-1 trial criteria. For all comparisons, statistical significance was accepted when $P<0.05$.

\section{Results}

Patient data

From the 123 patients aged over 60 who underwent elective EVAR with a bifurcated stent, 61 patients had aortic anatomy which would have allowed them to be entered into the EVAR-1 Trial, whilst 62 patients failed to meet one or more of the entrance criteria (Table 1). Between the two groups, patients were of a similar age [74.0 $00.8(61-68)$ vs $74.8 \pm 0.4$ (64-68) years] and had a similar AAA diameter [6.4 $\pm 0.1(5.5-8.7)$ vs $6.2 \pm 0.1(5.5-8.6) \mathrm{cm}]$. In both groups there was a similar overall male gender bias (male:female ratio was 58:3 vs 57:5).

Fluoroscopic screening time and radiation burden from EVAR

Patients who failed to meet the EVAR-1 trial criteria had a

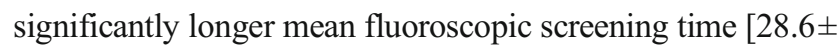
1.8 (10.1-78.0) vs $22.4 \pm 1.3(8.4-56.7) \mathrm{min}]$ associated with a significantly higher mean radiation dose [DAP: $134.6 \pm 9.6$ (34.8-459.7) vs 90.7 $\pm 5.5(11.0-190.8)$ Gy.cm² ; ED: $25.3 \pm 1.8$ (6.5-86.5) vs $17.1 \pm 1.0(2.1-35.9) \mathrm{mSv}$; Fig. 1].

Radiation burden from additional radiological examinations

Of the 123 patients selected for analysis, 11 patients underwent pre-EVAR iliac artery embolisation with an average radiation dose (DAP) of $137.2 \pm 24.3 \mathrm{~Gy} . \mathrm{cm}^{2}$ and an ED of $25.8 \pm$ $4.6 \mathrm{mSv}$. Of these, four patients met the criteria whilst seven patients did not meet the criteria for the EVAR-1 trial.

Using our CT aorta protocol, the radiation dose (DLP) from our 16-slice CT system was $696 \mathrm{mGy} . \mathrm{cm}$ and from our 64-slice CT system was 1137 mGy.cm, giving an average radiation dose for a $\mathrm{CT}$ aorta in our institution at 916.5 mGy.cm (DLP) or $16.0 \mathrm{mSv}$ (ED).

Hence, if a patient failed to meet the EVAR-1 trial criteria, they could potentially receive as much $99.1 \mathrm{mSv}$ of radiation within their first year as a result of routine CT examinations $(1 \times$ pre-EVAR and $2 \times$ post-EVAR $)$, the EVAR procedure itself and from a pre-EVAR iliac artery embolisation (Table 2).

\section{Discussion}

The EVAR-1 trial demonstrated a significant reduction in 30-day post-operative morbidity and mortality in patients undergoing EVAR compared with open aneurysm repair [1, 3]. However, this trial only selected patients based on

Table 1 Patient data

\begin{tabular}{lll}
\hline & $\begin{array}{l}\text { Patients who fit the } \\
\text { EVAR-1 trial criteria }\end{array}$ & $\begin{array}{l}\text { Patients who do not fit } \\
\text { the EVAR-1 trial criteria }\end{array}$ \\
\hline $\begin{array}{c}\text { Number (male: } \\
\text { female ratio) }\end{array}$ & $61(58: 3)$ & $62(57: 5)$ \\
$\begin{array}{l}\text { Age (years) } \\
\text { Aneurysm } \\
\text { diameter }(\mathrm{cm})\end{array}$ & $74.0 \pm 0.8$ & $74.8 \pm 0.4$ \\
\hline
\end{tabular}



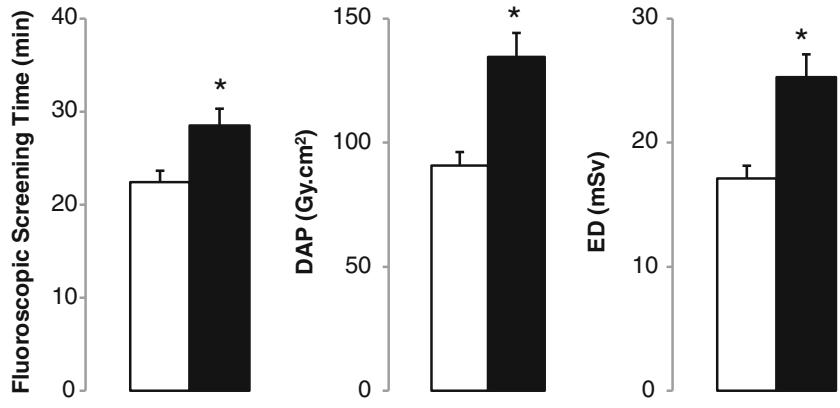

Fig. 1 The fluoroscopic screening time, DAP and ED for EVAR procedures. Bars represent the mean \pm SEM for the fluoroscopic screening time ( $\mathrm{min}$ ), dose area product (DAP; Gy.cm2) and effective dose (ED; mSv). White barpatients who fit the EVAR-1 trial criteria; black barpatients who do not fit the EVAR-1 trial criteria. Significant differences: ${ }^{*} P<0.05$ (unpaired $t$-test)

specific anatomical criteria, age and physical health. Nevertheless, over the past few years these results have been used to justify performing more complicated EVAR procedures in patients with more challenging aortic anatomy; however, this has not been taken into account when previous studies have determined the average dose of radiation from an EVAR procedure [8-10]. Furthermore, clinicians are becoming increasingly concerned as to the risks that patients may encounter based on the radiation dose from EVAR and its associated pre- and post- procedural investigations. Hence, the present study was undertaken to address these questions.

Our results show there is indeed a difference in the radiation dose depending on the complexity of the EVAR procedure. Patients who failed to meet the EVAR-1 trial criteria had EVAR procedures with longer fluoroscopic screening times associated with higher radiation doses. However, the radiation dose from an EVAR procedure alone, even in complicated cases, is not as high even when compared with other interventional procedures [6, 7]. Hence, taken on their own the radiation risks from EVAR are not high enough to affect the benefit of the reduced morbidity and mortality offered by EVAR compared with open aneurysm repair.
Of more concern is the additional increase in the radiation burden when the first year $\mathrm{CT}$ examinations are taken into consideration. As part of the EVAR-1 trial protocol, every patient also received one CT examination at 2 months pre-EVAR, to plan the procedure based on the anatomy of their aorta, and two surveillance CT examinations at 6 weeks and 12 months post-EVAR, to determine any complications following aortic stent placement. Based on our department's CT protocols, the mean radiation received from a CT aorta examination was $916.5 \mathrm{mGy} . \mathrm{cm}$. Hence, if a patient receives three CT examinations within the first year, this amounts to 2,749.5 mGy.cm. When converted to an effective dose, this works out as $48 \mathrm{mSv}$ of radiation, which is a considerable dose, especially with respect to the average yearly background dose of only $2.4 \mathrm{mSv}$. In addition, if a patient also requires a pre-EVAR iliac artery embolisation, then the mean radiation dose received from this intervention was $25.8 \mathrm{mSv}$. Surprisingly, this intervention delivered a greater dose of radiation to the patient than an EVAR procedure itself, probably due to the repeated fluoroscopic sequences often with oblique imaging, which need to be undertaken to complete the embolisation procedure. If this dose is also taken into account, then the radiation burden to patients can be fairly considerable within the first year. Although many patients who undergo open aneurysm repair will also require at least a pre-operative CT for planning and possibly even an early post-operative CT for evaluation of potential intraabdominal complications, EVAR patients will in reality require yearly CT examinations for lifelong surveillance of their aortic stents, further exposing them to radiation doses which will increase their lifetime risk of developing a radiation-induced cancer.

In addition, up to $41 \%$ of EVAR patients have been shown to develop post-procedural complications, with $20 \%$ subsequently requiring fluoroscopically based radiological re-intervention [1]. These percentages will also most likely increase for patients who failed to meet the EVAR-1 trial criteria, as their procedures will inevitably be more complicated. In these circumstances, the radiation burden

Table 2 The average radiation burden for EVAR procedures, CT aorta examinations and Iliac artery embolisation. Values are the mean doses for each examination

\begin{tabular}{|c|c|c|c|c|c|c|}
\hline & \multicolumn{3}{|c|}{ Patients who fit the EVAR-1 trial criteria } & \multicolumn{3}{|c|}{ Patients who do not fit the EVAR-1 trial criteria } \\
\hline & DAP $\left(\mathrm{Gy} . \mathrm{cm}^{2}\right)$ & DLP (mGy.cm) & $\mathrm{ED}(\mathrm{mSv})$ & DAP $\left(\mathrm{Gy} \cdot \mathrm{cm}^{2}\right)$ & DLP (mGy.cm) & $\mathrm{ED}(\mathrm{mSv})$ \\
\hline EVAR procedure alone & 90.7 & & 17.1 & 134.6 & & 25.3 \\
\hline CT aorta & & 916.5 & 16.0 & & 916.5 & 16.0 \\
\hline Iliac artery embolization (IAE) & 137.2 & & 25.8 & 137.2 & & 25.8 \\
\hline EVAR procedure $+3 \times \mathrm{CT}$ aorta & & & 65.1 & & & 73.3 \\
\hline EVAR procedure $+3 \times \mathrm{CT}$ aorta $+\mathrm{IAE}$ & & & 90.9 & & & 99.1 \\
\hline
\end{tabular}


may start to approach values which may justifiably raise the concern regarding the benefits of EVAR, especially in patients who (1) are younger, (2) require significantly longer procedures, such as those involving fenestrated or branched grafts (both of which were not included in the analyses of the present paper), or (3) require a second or even third EVAR to correct a persistent endoleak or technical problem such as limb dislocation or graft migration.

As the radiation from repetitive CT examinations contributes to a significant proportion of the radiation burden in patients undergoing EVAR, other imaging investigations which do not use ionising radiation should be considered for routine surveillance of aortic grafts, monitoring aneurysm size and detecting endoleaks in order to reduce the radiation burden to patients. These include colour Doppler ultrasound (CDUS), contrast-enhanced ultrasound (CEUS) and magnetic resonance angiography (MRA) [14-16]. Recently, Cantisani et al. [15] have shown that the accuracy of CEUS in detecting endoleaks is higher than CDUS and similar to CT and MRA. Furthermore, CEUS was noted to be a feasible tool in the long-term surveillance after EVAR and may better classify enoleaks missed by other imaging modalities. MRA with the blood-pool contrast agents has also been shown to have a higher diagnostic accuracy in the detection of endoleaks compared with CT [16, 17]. Although MRA can suffer from susceptibility artifact from metallic objects, most endografts now use metallic endoskeletons or exoskeletons that are not made from stainless steel and hence do not interfere with diagnostic images produced by MRA. With the knowledge of the high radiation penalty incurred by $\mathrm{CT}$ in the follow-up of EVAR patients, the current clinical practice in most departments, including our own, is to now use non-ionising radiation based imaging modalities, reserving the use of $\mathrm{CT}$ for complicated cases.

It is difficult to draw any firm conclusions regarding the actual risk from the radiation given to patients during EVAR or any other interventional procedure that uses fluoroscopic guidance. Several studies have attempted to assign cancer-related risks to interventional procedures using the BEIR VII report [18]. The BEIR VII report is based primarily on epidemiologic studies of survivors of the atomic bombs using organ doses and estimates the risk of developing cancer per unit of radiation exposure based on populations exposed to elevated levels of radiation [19]. Using data from the BEIR VII report assumes a linear dose-response relationship to the lower dose rate of exposure in medical procedures. Hence, these risks are more appropriately applied to patients who are uniformly irradiated, which is not the case for fluoroscopic and CT examinations where specific areas of interest are only exposed to a radiation field. Indeed, models that use individual organ doses have been shown to generate considerably higher radiationinduced cancer risks. Furthermore, it would be difficult to apply our data to such models as the dynamic nature of the fluoroscopic examination during EVAR means that we would be unable to assign any single organ with an accurate dose based on the total radiation dose recorded for the entire procedure. It is also important to appreciate that there may be some bias from radiation absorbed by the table, padding and variations of the tube angle and patient size. In addition, as the majority of the patients undergoing EVAR are elderly and often with multiple comorbidities, their lifetime risk of developing cancer secondary to radiation exposure is likely to be sufficiently low as to allow them to benefit from EVAR when compared with the higher complication risks associated with complicated open surgery and general anaesthesia.

In summary, uncomplicated EVAR procedures have lower fluoroscopic screening times and deliver acceptable doses of radiation to patients, supporting their use over open aneurysm repair in appropriate patients. The radiation burden significantly increases in patients who require preEVAR iliac artery embolisation and when routine surveillance CT examinations are taken into consideration. Undertaking EVAR in younger patients should be viewed with caution as these patients have a greater lifetime risk of developing a radiation-induced cancer. Furthermore, as repetitive CT examinations have been shown to cumulatively deliver high doses of radiation to patients, this highlights the importance of following-up patients with non-ionising radiation techniques whenever possible. More complicated EVAR procedures in patients with less favorable aortic anatomy must be planned with caution, as they will deliver larger doses of radiation. Furthermore, these procedures are often associated with additional reintervention and require more frequent follow-up CT examinations, ultimately increasing a patient's potential radiation-induced cancer risk. The results of the present study highlight the issues of radiation dose associated with EVAR procedures and related interventions, thereby allowing clinicians to provide patients with the appropriate information and counseling regarding potential levels of exposure. In addition, these findings will help clinicians to provide better informed consent, especially for those patients requiring more complicated EVAR procedures.

Acknowledgments The authors would like to thank Professor John Boice, Professor of Epidemiology, International Epidemiology institute, Rockville, MD, USA and Member of the International Committee on Radiation Protection for his advice on the preparation of the this manuscript.

Conflict of interest The authors declare that they have no conflicting interests. 


\section{References}

1. Greenhalgh RM, Brown LC, Kwong GP, Powell JT, Thompson SG (2004) Comparison of endovascular aneurysm repair with open repair in patients with abdominal aortic aneurysm (EVAR trial 1), 30-day operative mortality results: randomised controlled trial. Lancet 364:843-848

2. Prinssen M, Verhoeven EL, Buth J, Cuypers PW, van Sambeek MR, Balm R, Buskens E, Grobbee DE, Blankensteijn JD, Dutch Randomized Endovascular Aneurysm Management (DREAM) Trial Group (2004) A randomized trial comparing conventional and endovascular repair of abdominal aortic aneurysms. N Engl J Med 351:1607-1618

3. EVAR Trial Participants (2005) Endovascular aneurysm repair versus open repair in patients with abdominal aortic aneurysm (EVAR trial 1): randomised controlled trial. Lancet 365:2179-2186

4. Miller DL, Balter S, Cole PE, Lu HT, Schueler BA, Geisinger M, Berenstein A, Albert R, Georgia JD, Noonan PT, Cardella JF, St George J, Russell EJ, Malisch TW, Vogelzang RL, Miller GL III, Anderson J, RAD-IR study (2003) Radiation doses in interventional radiology procedures: the RAD-IR study: part I: overall measures of dose. J Vasc Interv Radiol 14:711-727

5. Hausler U, Czarwinski R, Brix G (2009) Radiation exposure of medical staff from interventional $\mathrm{x}$-ray procedures: a multicentre study. Eur Radiol 19:2000-2008

6. Bor D, Olgar T, Toklu T, Caglan A, Onal E, Padovani R (2009) Patient doses and dosimetric evaluations in interventional cardiology. Phys Med 25:31-42

7. Brambilla M, Marano G, Dominietto M, Cotroneo AR, Carriero A (2004) Patient radiation doses and references levels in interventional radiology. Radiol Med 107:408-418

8. Geijer H, Larzon T, Popek R, Beckman KW (2005) Radiation exposure in stent-grafting of abdominal aortic aneurysms. Br J Radiol 78:906-912

9. Weerakkody RA, Walsh SR, Cousins C, Goldstone KE, Tang TY, Gaunt ME (2008) Radiation exposure during endovascular aneurysm repair. Br J Surg 95:699-702

10. Kalef-Ezra JA, Karavasilis S, Ziogas D, Dristiliaris D, Michalis LK, Matsagas M (2009) Radiation burden of patients undergoing endovascular abdominal aortic aneurysm repair. J Vasc Surg 49:283-287
11. Mills JL Sr, Duong ST, Leon LR Jr, Goshima KR, Ihnat DM, Wendel CS, Gruessner A (2008) Comparison of the effects of open and endovascular aortic aneurysm repair on long-term renal function using chronic kidney disease staging based on glomerular filtration rate. J Vasc Surg 47:1141-1149

12. Diehm N, Tsoukas AI, Katzen BT, Benenati JF, Baum S, Pena C, Dick F (2008) Matched-pair analysis of endovascular versus open surgical repair of abdominal aortic aneurysms in young patients at low risk. J Vasc Interv Radiol 19:645-651

13. Huda W, Ogden KM, Khorasani MR (2008) Converting doselength product to effective dose at CT. Radiology 248:995-1003

14. Collins JT, Boros MJ, Combs K (2007) Ultrasound surveillance of endovascular aneurysm repair: a safe modality versus computed tomography. Ann Vasc Surg 21:671-675

15. Cantisani V, Ricci P, Grazhdani H, Napoli A, Fanelli F, Catalano C, Galati G, D'Andrea V, Biancari F, Passariello R (2011) Prospective comparative analysis of colour-Doppler ultrasound, contrast-enhanced ultrasound, computed tomography and magnetic resonance in detecting endoleak after endovascular abdominal aortic aneurysm repair. Eur J Vasc Endovasc Surg 41:186-192

16. Wieners G, Meyer F, Halloul Z, Peters N, Rühl R, Dudeck O, Tautenhahn J, Ricke J, Pech M (2010) Detection of type II endoleak after endovascular aortic repair: comparison between magnetic resonance angiography and blood-pool contrast agent and dual-phase computed tomography angiography. Cardiovasc Intervent Radiol 33:1135-1142

17. Cornelissen SA, Prokop M, Verhagen HJ, Adriaensen ME, Moll FL, Bartels LW (2010) Detection of occult endoleaks after endovascular treatment of abdominal aortic aneurysm using magnetic resonance imaging with a blood pool contrast agent: preliminary observations. Invest Radiol 45:548-553

18. National Research Council (2006) Health risks from exposure to low levels of ionizing radiation: BIER VII Phase 2, Committee to Assess Health Risks from Exposure to Low Levels of Ionizing Radiation, Board on Radiation Effects Research, Division on Earth and LifeSciences. The National Academies Press, Washington

19. Vijayalakshmi K, Kelly D, Chapple CL, Williams D, Wright R, Stewart MJ, Hall JA, Sutton A, Davies A, Haywood J, de Belder MA (2007) Cardiac catheterisation: radiation doses and lifetime risk of malignancy. Heart 93:370-371 